\title{
Factors associated with maintenance of antibody responses to influenza vaccine in older, community-dwelling adults
}

\author{
H Keipp Talbot ${ }^{*}$, Laura A Coleman², Yuwei Zhu', Sarah Spencer ${ }^{3}$, Mark Thompson $^{3}$, Po-Yung Cheng ${ }^{3}$, \\ Maria E Sundaram ${ }^{4}$, Edward A Belongia ${ }^{5}$ and Marie R Griffin ${ }^{1}$
}

\begin{abstract}
Background: Little is known about factors associated with maintenance of hemagglutinin inhibition (HAl) antibodies after influenza vaccination in older adults.

Methods: Adults $\geq 50$ years of age were vaccinated prior to the 2009-10 influenza season. Serum was drawn pre-vaccination (S1), 21-28 days post-vaccination (S2), and after the influenza season (S3) for HAl assays. Seroconversion was defined as $\geq 4$-fold increase $S 1$ to $S 2$ (or if $S 1<10$, by an $S 2 \geq 40$ ) and seroprotection was defined as $S 2 \geq 40$. Maintenance of antibody response was measured in participants with an $\mathrm{S} 2 \geq 40$, and defined as an $\mathrm{S} 3 \geq 40$.

Results: We enrolled 510 participants during Fall 2009 at Vanderbilt University Medical Center and Marshfield Clinic Research Foundation. Participants' mean age was 64 years with $62 \%$ female and $96 \%$ white. Seroconversion and seroprotection rates were lowest for influenza A H1N1 (12\% and 26\%, respectively), highest for influenza A H3N2 ( $45 \%$ and $82 \%$ ), and intermediate for influenza B (28\% and $72 \%$ ). Of the participants with an S2 $\geq 40,36 \%(46 / 126), 71 \%$ (289/407), and 74\% (263/354) maintained an S3 $\geq 40$ for H1N1, H3N2, and B influenza vaccine strains, respectively. S1 $\mathrm{HAl}$ titer was strongly associated with both post-vaccination seroprotection and maintaining seroprotection at S3 for all three influenza antigens. Age, sex, body mass index, self-reported stress, and vaccination site were not consistently associated with vaccine response or maintenance of response.
\end{abstract}

Conclusions: Pre-vaccination antibody titer was the only study variable consistently and positively associated with both serologic response to vaccination and maintenance of response. Antibody responses were lowest for the H1N1 vaccine strain.

ClinicalTrials: gov Identifier: NCT02401893

Keywords: Influenza, Older adults, Immunogenicity, Vaccine

\section{Background}

In the United States, yearly influenza vaccination begins in August or September. However, influenza season can extend into April of the following year. The duration of protection from annual vaccination in older adults is unknown, and little is known about factors associated with the maintenance of response throughout the influenza season. This study evaluated factors associated with the immune response of older adults to trivalent influenza

\footnotetext{
* Correspondence: Keipp.talbot@vanderbilt.edu

'Vanderbilt University Medical Center, A2200 MCN 1161 21st Ave, Nashville, TN 37232, USA

Full list of author information is available at the end of the article
}

vaccine and the maintenance of antibody responses for the duration of the influenza season.

\section{Methods}

Subjects

Subjects were enrolled at two sites, Vanderbilt University Medical Center (Nashville, TN) and Marshfield Clinic Research Foundation (Marshfield, WI), during September and October 2009. Subjects were eligible for recruitment if they were $\geq 50$ years of age and had no contraindication to influenza vaccination. Subject recruitment included advertisements at Vanderbilt University Medical Center and letters of invitation to older adults who had received an 
influenza vaccine in the year prior at Marshfield Clinic [1]. All subjects were vaccinated either by their usual caregiver or by the study staff. Strain components for the 20092010 Northern Hemisphere vaccine included A/Brisbane/ 59/2007-like (H1N1), A/Brisbane/10/2007-like (H3N2), and $\mathrm{B} / \mathrm{Brisbane} / 60 / 2008$-like. Participants were given the trivalent seasonal vaccine because the $2009 \mathrm{H} 1 \mathrm{~N} 1$ pandemic vaccine was not available.

\section{Data collection}

All subjects donated serum pre-vaccination (S1) during September through October 2009, 21-28 days postvaccination (S2) and post-influenza season, May through July, 2010, approximately 250 days (8 months) postvaccination (S3). We chose the post-influenza season blood draw to be 8 months following S1 since this is likely to be the maximal duration of needed protection for a given influenza season. In the US, some influenza vaccines are now being given in August, and it is not unusual for the influenza season to extend into March and April. Study procedures, informed consent documents and data collection forms were reviewed and approved by Institutional Review Boards at each of the study sites.

Age, co-morbid conditions, sex, and race were ascertained from participant interview. Recent chemotherapy, radiation therapy, or use of immunomodulating medications were ascertained by self-report or chart review. CDC-defined high risk medical conditions were identified by self-report of organ transplantation, cancer, diabetes mellitus, splenectomy (functional or anatomic), cardiovascular disease, renal disease, sickle cell disease, chronic pulmonary disease, seizure disorder, immune deficiency, or dementia [2]. Self-reported stress was determined by asking participants to respond yes or no to the question "Have you suffered psychological stress or acute disease in the past 3 months?" included as part of the Mini Nutritional Assessment Questionnaire [3]. Height and weight were measured by research study staff and body mass index (BMI) was calculated as weight $(\mathrm{kg}) \div$ height $\left(\mathrm{m}^{2}\right)$. Study participants completed the Vulnerable Elders Survey (VES-13) which is a series of questions to determine risk for health deterioration. The scale ranges from 0 to 10 , where a participant with a score of 3 or greater is considered vulnerable and with 10 being most vulnerable [4].

\section{Laboratory methods}

Blood samples were processed, stored, and shipped by each institution's local Sample Processing Core to Battelle (Columbus, $\mathrm{OH}$ ). Hemagglutinin inhibition (HAI) testing was performed in duplicate against the influenza vaccine strains in the 2009-2010 Northern Hemisphere influenza vaccine. Although there is debate about the best correlate of protection for influenza [5], seroprotection was defined as an HAI titer of $\geq 40$ since it is the correlate recognized by the United States Food and Drug Administration [6]. Seroconversion was measured at S2 and defined as a four-fold rise in HAI post-influenza vaccination compared to pre-vaccination or $\geq 40$ if $\mathrm{S} 1$ was $<10$. Maintenance of antibody response was measured in participants with an $S 2 \geq 40$, and defined as an $S 3 \geq 40$. If duplicate HAI results were discrepant by more than two fold, a third test was performed and the minimum result was recorded.

\section{Statistical analysis}

Multivariable logistic regression models were run for the binary outcomes seroprotection at S2 and S3 and seroconversion at S2. Age, BMI, stress, high risk medical conditions (yes/no), female gender, study site and transformed S1 titer were included in all models. Time in days from S1 to S3 was included in models with S3 related outcomes as a continuous variable. Restricted cubic splines were applied to age and BMI. All raw HAI titers were $\log$ transformed using the method outlined by Beyer [7], changing dilution titers to integers with HAI $<10$ coded as 0,10 as 1,20 as 2, 40 as 3 and so on. Interaction terms between age and high risk status, and stress or age and sex were tested and were not included based on non-significant $\mathrm{p}$ values of overall interaction terms. Figures were generated by predicting the probability of having a HAI titer $\geq 40$ by logistic regression. All analyses were done using $\mathrm{R}$ version 2.12.2.

\section{Results}

A total of 510 participants were enrolled during September and October of 2009 at Vanderbilt University Medical Center (259) and Marshfield Clinic Research Foundation (251). The mean age was 64 years (Interquartile Range [IQR]: 58,74$)$ with $62 \%$ female and $96 \%$ white. The participants were very functional with only $9.4 \%$ having a VES score of $\geq 3$ and the remaining $91.6 \%$ having a score $<3$. The 494 participants who completed all three visits were similar to the total enrolled; $37 \%$ had a high risk medical condition, median BMI was $29 \mathrm{~kg} / \mathrm{m}^{2}$ (interquartile range [IQR]: 25,34 ), and $9 \%$ reported having a stressful event in the last 12 months. The median duration between first and third study visit was 257 days (IQR: 250, 263). Data on immunization within the past 3 years were available at Marshfield Clinic; $99 \%$ of participants were vaccinated at least once in the previous three years.

Pre-vaccination geometric mean S1 titers for H1N1, $\mathrm{H} 3 \mathrm{~N} 2$, and B were $7.65,27.50$, and 24.72 respectively. Increasing age was associated with significantly decreased odds of baseline seroprotection for H1N1 ( $<<0.01$ ), but not for H3N2 or influenza B. Female sex was associated with decreased odds of seroprotection for influenza B (0.62, 95\% CI: $0.41,0.92)$, but not for the other strains. 
Post-vaccination responses to H1N1 were the lowest of the three tested strains, with only $12 \%$ and $26 \%$ of participants achieving seroconversion and seroprotection at S2, respectively. Seroconversion and seroprotection were highest for $\mathrm{H} 3 \mathrm{~N} 2$ (45\% and $82 \%$, respectively; Table 1 ). Table 2 summarizes the characteristics of participants that achieved seroprotection post-vaccination. There were few prior differences between those who did and did not attain seroprotection at S2 (Table 3). Participants at Vanderbilt were significantly less likely to achieve seroprotection than participants at the Marshfield Clinic for HIN1 and B vaccine strains. Results of multivariable logistic regression models using seroprotection at 28 days post-vaccination as the outcome are shown in Table 3 (binomial variables) and Figure 1 (continuous variables). BMI was not included in the figure since it was not statistically significantly associated with seroprotection for $\mathrm{H} 1 \mathrm{~N} 1$ or H3N2 ( $\mathrm{p}=1.0, \mathrm{p}=0.9$ ). Pre-vaccination HAI titer was the only consistent predictor of post-vaccination seroprotection (Figure 1). In sensitivity analyses, excluding all participants with an $\mathrm{S} 1 \geq 40$ prior to vaccination, pre-vaccination HAI titer remained strongly associated with seroprotection at S2 $(\mathrm{p}<0.0001)$ for all three antigens. In this sensitivity analysis, female sex was also associated with seroprotection at S2 for H1N1 $(p=0.0004)$ and H3N2 ( $\mathrm{p}=0.04)$.

Maintenance of antibody response was assessed in participants who had an S2 HAI titer of $\geq 40$ (Table 4). Maintenance of a protective antibody response was defined by an $\mathrm{S} 3 \geq 40$. Of the participants with an $\mathrm{S} 2 \geq 40,36 \%$ (46/ 126), $71 \%$ (289/407), and $74 \%$ (263/354) maintained an $\mathrm{S} 3 \geq 40$ for $\mathrm{H} 1 \mathrm{~N} 1, \mathrm{H} 3 \mathrm{~N} 2$, and B influenza vaccine strains, respectively. S1 HAI titer was strongly and consistently associated with maintenance of seroprotection (Figure 2). Higher stress was modestly associated with maintenance of seroprotection for the B strain only (Table 4).

\section{Discussion}

In our cohort of adults $\geq 50$ years of age, the initial antibody response to influenza vaccination and maintenance

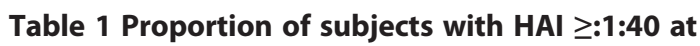
baseline (S1), 28 days post-vaccination (S2), and after influenza season (S3) and with seroconversion at S2, by influenza strain

\begin{tabular}{llll}
\hline Seroprotection & H1N1 & H3N2 & B \\
& $\%(\mathbf{N})$ & $\%(\mathbf{N})$ & $\%(\mathbf{N})$ \\
\hline Baseline (S1) & $9 \%(44)$ & $48 \%(235)$ & $43 \%(214)$ \\
28 days post-vaccination (S2) & $26 \%(126)$ & $82 \%(407)$ & $72 \%(354)$ \\
After influenza season (S3) & $9 \%(46)$ & $59 \%(289)$ & $53 \%(263)$ \\
Seroconversion & & & \\
28 days post-vaccination (S2) & $12 \%(60)$ & $45 \%(222)$ & $28 \%(138)$ \\
\hline
\end{tabular}

Table 2 Enrolled subjects with post-vaccination (S2) titers $\geq 1: 40$

\begin{tabular}{|c|c|c|c|}
\hline & $\begin{array}{l}\text { H1N1 } \\
(\mathrm{N}=126) \\
\%(n)\end{array}$ & $\begin{array}{l}\text { H3N2 } \\
(\mathrm{N}=407) \\
\%(n)\end{array}$ & $\begin{array}{l}B \\
(N=354) \\
\%(n)\end{array}$ \\
\hline Age (Years) & $62(57,74)$ & $65(58,75)$ & $66(58,76)$ \\
\hline Sex (Female) & $71 \%(90)$ & $64 \%(260)$ & $61 \%(215)$ \\
\hline Race (White) & $96 \%(121)$ & $96 \%(390)$ & $96 \%(341)$ \\
\hline $\begin{array}{l}\text { Recent illness or stressor } \\
\text { (Yes) }\end{array}$ & $32 \%(40)$ & $35 \%(143)$ & $39 \%(139)$ \\
\hline Stress (Yes) & $10 \%(12)$ & $8 \%(34)$ & $9 \%(32)$ \\
\hline $\begin{array}{l}\text { Time from S1 to S3 (days) } \\
\text { (mean, IQR) }\end{array}$ & $257(251,266)$ & $257(250,264)$ & $257(251,264)$ \\
\hline
\end{tabular}

of seroprotective levels of antibody response throughout the influenza season were strongly associated with prevaccination antibody levels for all three vaccine strains.

A number of prior studies have assessed the duration of antibody response in older adults, but few have determined factors associated with maintenance of protection [8-17]. Prior studies have been inconsistent about duration of protection with results ranging from $15 \%$ maintaining seroprotection at 5 months [8] to $88 \%$ maintaining seroprotection at 12 months [16]. Some studies showing prolonged maintenance may have been complicated by interval natural infection. Our results are unlikely to have been confounded by natural influenza infection since the only influenza virus strain circulating was the pandemic strain, not yet included in the seasonal trivalent influenza vaccine.

A study in Korean adults $\geq 65$ years of age was one of the few prior studies to assess factors associated with maintenance of antibody levels. Similar to our results, maintenance of antibody response was associated with pre-vaccination HAI titer $\geq 40$. In this population, maintenance was also associated with less advanced age [18]. This latter difference in findings could be due to the older age of participants

Table 3 Baseline characteristics associated with seroprotection post-vaccination (S2) versus not achieving seroprotection

\begin{tabular}{|c|c|c|c|c|c|c|}
\hline \multirow{2}{*}{$\begin{array}{l}\text { Baseline } \\
\text { characteristics }\end{array}$} & \multicolumn{2}{|l|}{ H1N1 } & \multicolumn{2}{|l|}{ H3N2 } & \multicolumn{2}{|l|}{ B } \\
\hline & $\begin{array}{l}\text { Odds } \\
\text { ratio* }\end{array}$ & $p$ value & $\begin{array}{l}\text { Odds } \\
\text { ratio* }\end{array}$ & $p$ value & $\begin{array}{l}\text { Odds } \\
\text { ratio* }\end{array}$ & $p$ value \\
\hline $\begin{array}{l}\text { Sex (Female vs } \\
\text { Male) }\end{array}$ & 3.5 & $<0.01$ & 2.0 & 0.02 & 1.2 & 0.56 \\
\hline $\begin{array}{l}\text { High risk medical } \\
\text { condition (Yes vs No) }\end{array}$ & 1.2 & 0.60 & 0.8 & 0.53 & 1.1 & 0.74 \\
\hline $\begin{array}{l}\text { Recent illness or } \\
\text { stressor (Yes vs No) }\end{array}$ & 0.5 & 0.71 & 0.8 & 0.58 & 0.8 & 0.60 \\
\hline $\begin{array}{l}\text { Site (Vanderbilt vs } \\
\text { Marshfield) }\end{array}$ & 0.4 & 0.01 & 1.0 & 0.96 & 0.5 & 0.01 \\
\hline
\end{tabular}

*Odds ratios all adjusted for variables in table plus age, BMI, and transformed S1 titer using multivariable logistic regression. 


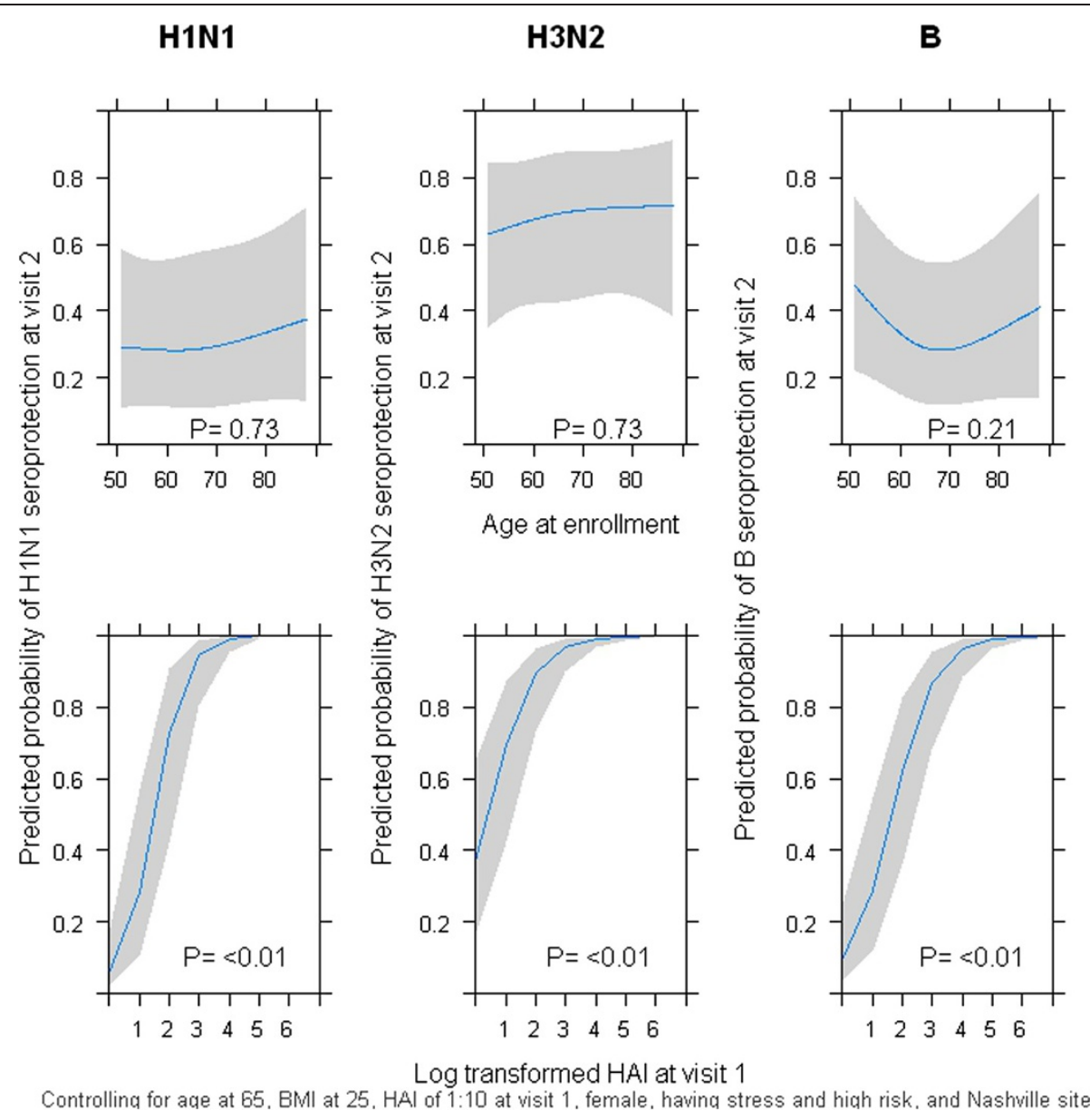

Figure 1 Predicted probability of seroprotection at S2 for each vaccine strain by age at enrollment and pre-vaccination HAl titer. Each graph shows the probability of seroprotection predicted by logistic regression models for H1N1 (first column), H3N2 (second column) and B (third column) by the age at enrollment (first row) or the pre-vaccination HAl titer (second row) 4 weeks post-vaccination.

(mean age $71.7 \pm 4.5$ ) in the Korean study compared to the mean age of $64 \pm 10.2$ years of our participants.

Interestingly, we also found differences between the two study sites. Participants at Vanderbilt were less likely to attain seroprotective levels of antibody to influenza A

Table 4 Baseline characteristics associated with maintaining versus not maintaining $\mathrm{HAI} \geq 1: 40$ at $\mathrm{S} 3$

\begin{tabular}{|c|c|c|c|c|c|c|}
\hline & \multirow{2}{*}{\multicolumn{2}{|c|}{$\frac{\mathrm{H} 1 \mathrm{~N} 1}{\mathrm{~N}=126}$}} & \multirow{2}{*}{\multicolumn{2}{|c|}{$\frac{\mathrm{H} 3 \mathrm{~N} 2}{\mathrm{~N}=407}$}} & \multirow{2}{*}{\multicolumn{2}{|c|}{$\frac{B}{N=354}$}} \\
\hline & & & & & & \\
\hline & $\begin{array}{l}\text { Odds } \\
\text { ratio* }\end{array}$ & p-value & $\begin{array}{l}\text { Odds } \\
\text { ratio* }\end{array}$ & p-value & $\begin{array}{l}\text { Odds } \\
\text { ratio* }\end{array}$ & p-value \\
\hline Sex (Female) & 1.2 & 0.77 & 1.1 & 0.70 & 0.8 & 0.46 \\
\hline High risk (Yes) & 1.4 & 0.57 & 1.7 & 0.12 & 0.9 & 0.70 \\
\hline $\begin{array}{l}\text { Recent Illness } \\
\text { or stressor (Yes) }\end{array}$ & 3.3 & 0.16 & 1.6 & 0.37 & 10.6 & 0.003 \\
\hline $\begin{array}{l}\text { Site (Vanderbilt } \\
\text { vs Marshfield) }\end{array}$ & 2.3 & 0.11 & 1.6 & 0.14 & 1.4 & 0.30 \\
\hline
\end{tabular}

*Odds ratios all adjusted for variables in table plus age, BMI, days between S3 and S1 and transformed S1titer using multivariable logistic regression.
H1N1 and influenza B, controlling for other factors. Age criteria for enrollment differed at the two sites, but these differences in attaining protective titers persisted after controlling for age. Participants at Marshfield Clinic were clinic patients recruited from those vaccinated the prior year; whereas participants at Vanderbilt were volunteers. It is possible that differences in vaccination or prior influenza disease not reflected in baseline S1 were responsible for differences observed. Differences were unlikely due to vaccination in the prior year since $>97 \%$ had received influenza vaccine in the year prior. Other explanations are differences in the specific vaccines administered or methods of administration, chance, or some other unmeasured factor.

Both sites had very low seroconversion rates to H1N1 and overall low seroprotection. Goodwin et al. summarized results from 31 studies of influenza vaccine responses in elderly adults 1986-2002, and reported an average seroconversion rate of $42 \%$ and a $69 \%$ seroprotection rate to H1N1 viruses [19]. However, there is considerable year to 


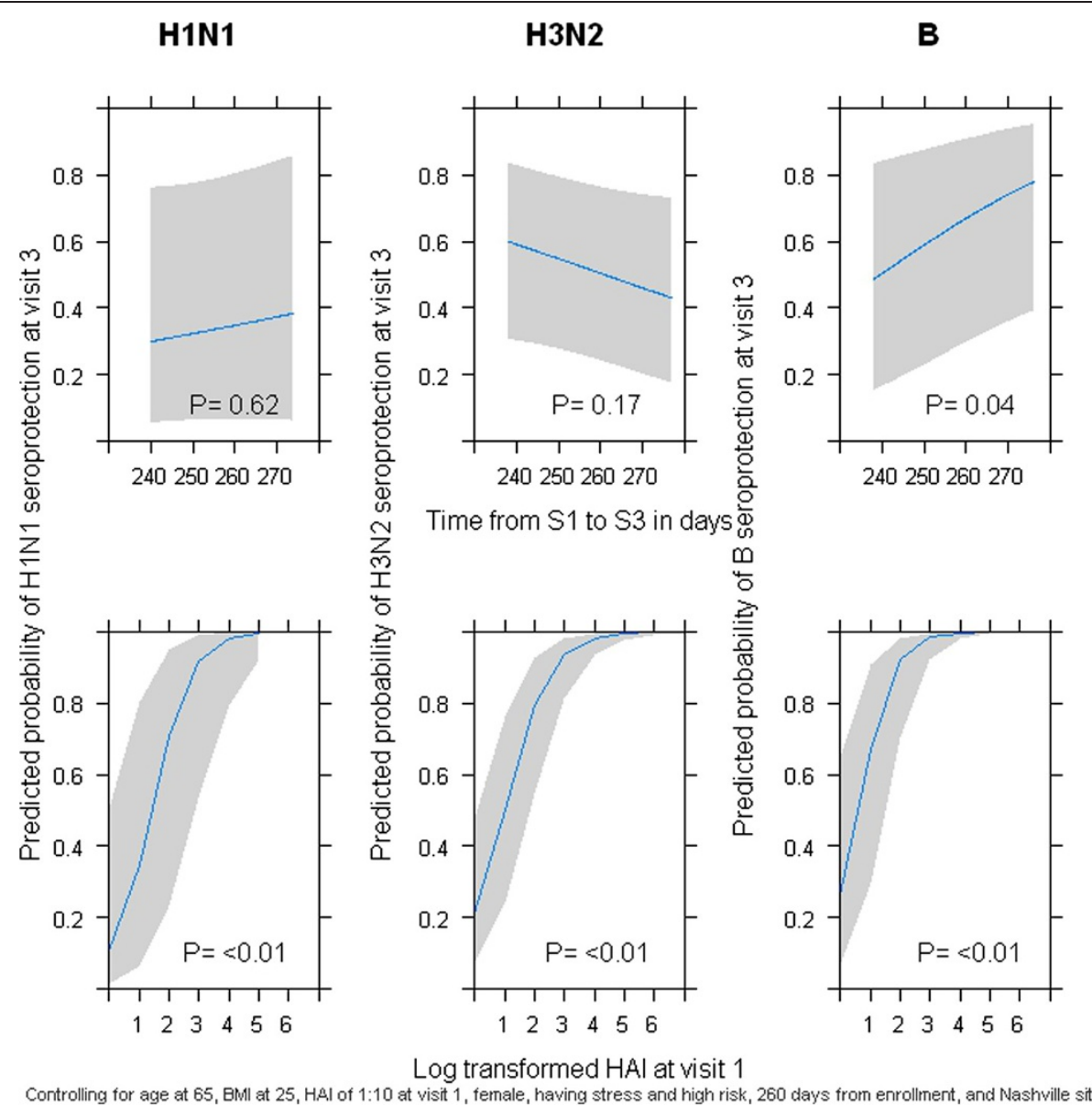

Figure 2 Predicted probability of seroprotection at $\mathrm{S} 3$ for each vaccine strain by age at enrollment and pre-vaccination HAI titer. Each graph shows the predicted probability of seroprotection for H1N1 (first column, H3N2 (second column) and B (third column) by the time from vaccination (first row) or the pre-vaccination HAl titer (second row) after influenza season for those with an HAl titer $\geq 40$ after immunization $(H 1 N 1, n=126$; $\mathrm{H} 3 \mathrm{~N} 2, \mathrm{n}=407$; and $\mathrm{B}: \mathrm{n}=354$ ).

year variability, and a seroprotection rate of $11 \%$ was reported in a study done during the 1993-1994 season among adults $\geq 65$ years of age when the $\mathrm{H} 1 \mathrm{~N} 1$ vaccine strain was A/Texas/36/91 [20].

This study was limited by several factors. Foremost, this is a single study year with a single seasonal influenza vaccine. Vaccine strains can change each year. In the 2009-2010 Northern Hemisphere influenza vaccine, both the $\mathrm{H} 1$ and the H3N2 components had been used during the prior years, but the $\mathrm{B}$ component for the 2009 vaccine was new. The repetitive use of an antigen may make pre-vaccination results more important than for a novel antigen. However even for the $\mathrm{B}$ antigen, prevaccination response remained the most prominent factor associated with vaccine response. Because most participants had been vaccinated within the past year, it was not possible to determine the effect of prior immunization. The results of this study may not be generalizable to very old adults, since the mean age was only 64 years of age and participants were generally healthy with $91.6 \%$ classified as not vulnerable. Lastly, an antibody titer of $\geq 40$ was chosen as the definition of seroprotection because this is the level of antibody used for influenza vaccine licensure [6]. It is unclear if this is a reliable predictor of protection in older adults. Even in younger adults, titers $\geq 40$ have been seen in cases of influenza vaccine failure [5].

The study clearly demonstrated that antibody response to trivalent inactivated influenza vaccine, and maintenance of this response, are associated with pre-vaccination antibody titers. Hence, older adults with low pre-vaccination HAI antibody titers are less likely to respond to influenza vaccination. It is unclear if these older adults are at higher risk for influenza and complications to influenza or if they may be vaccine failures. Future research will need to determine if this places these adults at higher risk, and whether specific types of vaccines will result in a more robust immune response and a greater likelihood of protection in these older adults. 


\section{Conclusions}

In summary, pre-vaccination antibody titer was the only study variable consistently and positively associated with both serologic response to vaccination and maintenance of response in older adults. Antibody responses were lowest for the $\mathrm{H} 1 \mathrm{~N} 1$ vaccine strain despite less severe disease in older adults due to H1N1 compared to other strains.

\section{Abbreviations}

BMI: Body mass index; CDC: Centers for Disease Control and Prevention; HAl: Hemagglutinin inhibition; IQR: Interquartile ratio.

\section{Competing interests}

H. Keipp Talbot has received research funding from Sanofi Pasteur, Medlmmune/Aztrazeneca and Giliead and is an advisor for Teva pharmaceuticals. Laura A. Coleman PhD, RD, currently works for Abbott Nutrition. At the time the study was conducted, she was at the Marshfield Clinic Research Foundation. Maria E. Sundaram, Edward A Belongia, and Marie Griffin have received research funding from Medlmmune. Yuwei Zhu, Sarah Spencer, Mark Thompson, Po-Yung Cheng, and David Shay have no conflicts of interest.

\section{Authors' contributions}

$H K T, L A C, M R G$, and EAB have made substantial contributions to conception and design, or acquisition of data, or analysis and interpretation of data; have been involved in drafting the manuscript or revising it critically for important intellectual content; and have given final approval of the version to be published; YZ, SS, MT, PC, and MES have made substantial contribution to the analysis and interpretation of data and have been involved in drafting the manuscript or revising it critically for important intellectual content; and have given final approval of the version to be published. All authors have read and approved the final manuscript.

\section{Acknowledgements}

We would like to thank all participants who donated their time and effort to participate in making this study possible.

\section{Funding}

This research was supported by: K23 Al074863-01A1 (PI, Talbot HK), CDC 1 U18 IP000184-01 (PI, Griffin MR), CDC 5 U18 IP000183-02 (PI, Belongia EA), CTSA award No. UL1TR000445 from the National Center for Advancing Translational Sciences and the Atlantic Philanthropies (USA) Inc, the Infectious Diseases Society of America, the John A. Hartford Foundation, Inc., and the Association of Specialty Professors.

The contents are solely the responsibility of the authors and do not necessarily represent official views of the Centers for Disease Control and Prevention and the National Center for Advancing Translational Sciences or the National Institutes of Health.

\section{Author details}

${ }^{1}$ Vanderbilt University Medical Center, A2200 MCN 1161 21st Ave, Nashville, TN 37232, USA. ${ }^{2}$ Abbott Nutrition, 3300 Stelzer Avenue, Columbus, $\mathrm{OH}$ 43219, USA. ${ }^{3}$ Centers for Disease Control and Prevention, 1600 Clifton Rd MS A32, Atlanta, GA 30333, USA. ${ }^{4}$ University of Minnesota, Minneapolis, MN 55455, USA. ${ }^{5}$ Marshfield Clinic Research Foundation, 1000 N. Oak Avenue, Marshfield, WI 54449, USA.

\section{Received: 6 November 2014 Accepted: 8 April 2015}

Published online: 23 April 2015

\section{References}

1. Talbot HK, Coleman LA, Crimin K, Zhu Y, Rock MT, Meece J. Association between obesity and vulnerability and serologic response to influenza vaccination in older adults. Vaccine. 2012;30:3937-43.

2. Grohskopf L, Uyeki T, Bresee J, Cox N. Prevention and control of influenza with vaccines: recommendations of the Advisory Committee on Immunization Practices (ACIP)-United States, 2012-13 influenza season. MMWR Morb Mortal Wkly Rep. 2012;61:613-8.

3. MNA Mini Nutritional Assessment. at http://mna-elderly.com.
4. Saliba D, Elliott M, Rubenstein LZ, Solomon DH, Young RT, Kamberg CJ, et al. The Vulnerable Elders Survey: a tool for identifying vulnerable older people in the community. J Am Geriatr Soc. 2001;49:1691-9.

5. Ohmit SE, Petrie JG, Cross RT, Johnson E, Monto AS. Influenza hemagglutination-inhibition antibody titer as a correlate of vaccine-induced protection. J Infect Dis. 2011;204:1879-85.

6. Guidance for Industry: Clincal Data Needed to Support hte Licensure of Seasonal Inactivated Influenza Vaccines 2007. (Accessed 9/9/14, at http://www.fda.gov/BiologicsBloodVaccines/GuidanceComplianceRegulatory Information/Guidances/Naccines/ucm074794.htm).

7. Beyer WE, Palache AM, Luchters G, Nauta J, Osterhaus AD. Seroprotection rate, mean fold increase, seroconversion rate: which parameter adequately expresses seroresponse to influenza vaccination? Virus Res. 2004;103:125-32.

8. Brydak LB, Machala M, Mysliwska J, Mysliwski A, Trzonkowski P. Immune response to influenza vaccination in an elderly population. J Clin Immunol. 2003;23:214-22.

9. Buxton JA, Skowronski DM, Ng H, Marion SA, Li Y, King A, et al. Influenza revaccination of elderly travelers: antibody response to single influenza vaccination and revaccination at 12 weeks. J Infect Dis. 2001;184:188-91.

10. Delafuente JC, Davis JA, Meuleman JR, Jones RA. Influenza vaccination and warfarin anticoagulation: a comparison of subcutaneous and intramuscular routes of administration in elderly men. Pharmacotherapy. 1998;18:631-6.

11. Hui SL, Chu LW, Peiris JS, Chan KH, Chu D, Tsui W. Immune response to influenza vaccination in community-dwelling Chinese elderly persons. Vaccine. 2006;24:5371-80.

12. Keylock KT, Lowder T, Leifheit KA, Cook M, Mariani RA, Ross K, et al. Higher antibody, but not cell-mediated, responses to vaccination in high physically fit elderly. J Appl Physiol. 2007;102:1090-8.

13. Mackenzie JS. Influenza subunit vaccine: antibody responses to one and two doses of vaccine and length of response, with particular reference to the elderly. Br Med J. 1977;1:200-2.

14. Minutello M, Senatore F, Cecchinelli G, Bianchi M, Andreani T, Podda A. Safety and immunogenicity of an inactivated subunit influenza virus vaccine combined with MF59 adjuvant emulsion in elderly subjects, immunized for three consecutive influenza seasons. Vaccine. 1999;17:99-104.

15. Mysliwska J, Trzonkowski P, Szmit E, Brydak LB, Machala M, Mysliwski A. Immunomodulating effect of influenza vaccination in the elderly differing in health status. Exp Gerontol. 2004;39:1447-58.

16. Praditsuwan $R$, Assantachai $P$, Wasi $C$, Puthavatana P, Kositanont U. The efficacy and effectiveness of influenza vaccination among Thai elderly persons living in the community. J Med Assoc Thai. 2005;88:256-64.

17. Van Hoecke C, Prikazsky V, Uto I, Menschikowski C. Immunogenicity of an inactivated split influenza vaccine in institutionalized elderly patients. Gerontology. 1996;42:190-8.

18. Song JY, Cheong HJ, Hwang IS, Choi WS, Jo YM, Park DW. Long-term immunogenicity of influenza vaccine among the elderly: Risk factors for poor immune response and persistence. Vaccine. 2010;28:3929-35.

19. Goodwin K, Viboud C, Simonsen L. Antibody response to influenza vaccination in the elderly: a quantitative review. Vaccine. 2006;24:1159-69.

20. Powers DC, Hanscome PJ, Pietrobon PJ. In previously immunized elderly adults inactivated influenza A ( $\mathrm{H} 1 \mathrm{~N} 1$ ) virus vaccines induce poor antibody responses that are not enhanced by liposome adjuvant. Vaccine. 1995;13:1330-5.

\section{Submit your next manuscript to BioMed Central and take full advantage of:}

- Convenient online submission

- Thorough peer review

- No space constraints or color figure charges

- Immediate publication on acceptance

- Inclusion in PubMed, CAS, Scopus and Google Scholar

- Research which is freely available for redistribution 